\title{
Diversidad y patrones de actividad de mamíferos medianos y grandes en la Reserva de la Biosfera La Encrucijada, Chiapas, México
}

\author{
Julio C. Hernández Hernández ${ }^{1}$, Cuauhtémoc Chávez ${ }^{2 *} \&$ Rurik List $^{2}$ \\ 1. Programa de Doctorado en Ciencias Biológicas y de la Salud, Universidad Autónoma Metropolitana-Cuajimalpa, \\ Vasco de Quiroga 4871, Col. Santa Fe Cuajimalpa, Ciudad de México 05348, México; biol.julio@gmail.com \\ 2. Área de Investigación en Biología de la Conservación, Departamento de Ciencias Ambientales, CBS Universidad \\ Autónoma Metropolitana-Lerma, Hidalgo Pte. 46, Col. La Estación Lerma, Estado de México 52006, México; \\ j.chavez@correo.ler.uam.mx, r.list@correo.ler.uam.mx \\ * Correspondencia
}

\author{
Recibido 20-X-2017. Corregido 18-I-2018. Aceptado 12-II-2018.
}

\begin{abstract}
Diversity and activity patterns of medium and large mammals in La Encrucijada Biosphere Reserve, Chiapas, Mexico. In Mexico, wetlands occupy an important portion of the country, among them, mangroves are particularly diverse. La Encrucijada Biosphere Reserve (ENBIRE) is a coastal ecosystem of mangrove located along the Pacific Coastal Plain in the Southwestern portion of Chiapas state. The ENBIRE mangroves are among the most important of the American Pacific Coast, highlighting the role they play in the maintenance of the community of mammals on the coast of the state. There are few studies in Mexico related to the mammals associated with these ecosystems, our aim was to estimate the diversity and activity patterns of medium and large mammals in the ENBIRE. Sampling was conducted from August 2015 to August 2016 using camera traps. We obteined 1851 independent photographs records, with a total sampling effort of 5400 trap-days. Nineteen species of mammals were registered belonging to 15 families and seven orders, and five species were new records to the area. Six species present are listed, two as endangered and four as threatened. Interpolation and extrapolation analyzes showed an asymptotic trend in the curves for the rainy and dry seasons. The coverage of the sample for both seasons was $99.9 \%$ and $99.8 \%$, respectively, indicating that both cases were a representative sample. According to the relative abundance index obtained, the most abundant species were Procyon lotor (IAR = 4.35), Nasua narica $(\mathrm{IAR}=3.91)$, Philander opossum $(\mathrm{IAR}=2.04)$, Cuniculus paca $(\mathrm{IAR}=1.89)$, Didelphis marsupialis $(\mathrm{IAR}=1.67)$ and Dasypus novemcinctus $(\mathrm{IAR}=1.02)$. In terms of patterns of activity C. paca, P. opossum, D. marsupialis and D. novemcinctus were primarily nocturnal; $P$. lotor showed a tendency towards nocturnal habits but was also recorded during the day; $N$. narica was mainly diurnal. This information can be useful to the creation of management programs and the conservation of medium and large mammals in the ENBIRE, especially for species at risk. Rev. Biol. Trop. 66(2): 634-646. Epub 2018 June 01.
\end{abstract}

Key words: camera-traps; species richness; wetlands; wild mammal ecology.

Los humedales se encuentran entre los ecosistemas de mayor importancia ecológica y económica del mundo (ten Brink et al., 2013); son indispensables debido a los innumerables servicios ecosistémicos que brindan, como el suministro de agua dulce, de alimentos, materiales de construcción y la mitigación del cambio climático (Mitsch, Gosselink, G., Anderson, \& Zhang, 2009). Ofrecen diversos hábitats que proveen alimento, refugio, áreas de reproducción y crianza para la fauna silvestre, manteniendo así una diversidad considerablemente mayor que otros ecosistemas (Ramsar, 2013). Son ambientes costeros altamente productivos y de protección del medio ambiente, debido a que actúan en el control de inundaciones, huracanes y efectos del oleaje, también son productores de oxígeno, capturan gases de efecto invernadero y son sumideros de bióxido de carbono (Troche-Souza et al., 2016). Sin embargo, la tasa actual de pérdida de este ecosistema es alta; cerca del $50 \%$ de 
ellos han desaparecido del planeta y existen regiones en las que el $99 \%$ se ha perdido (Van der Valk, 2006), particularmente por las actividades antropogénicas, donde la destrucción del hábitat, la contaminación y la sobreexplotación de los recursos son las actividades que más impactan (Parks \& Bonifaz, 1994; Mackey \& Smail, 1995; Rodríguez-Zúñiga et al., 2013).

En México, los humedales incluyen manglares, marismas, selvas y palmares inundables, popales y tulares, entre otros, y constituyen una superficie importante dentro del territorio nacional. De acuerdo con el inventario de humedales del 2012, México tiene 6331 sistemas de humedales que abarcan 9924 624 ha, lo que representa el $5 \%$ del territorio nacional (SEMARNAT, 2014). Entre ellos, los manglares ocupan un lugar privilegiado por la riqueza natural que encierran y los servicios ambientales que prestan (Spalding, Kainuma, \& Collins, 2010).

La superficie estimada de manglar en México para el año 2015 era de 775555 ha, donde un $63 \%$ se localiza dentro de Áreas Naturales Protegidas (ANP's) federales y estatales (Troche-Souza et al., 2016). Dentro de estas ANP's se encuentra La Reserva de la Biósfera La Encrucijada (REBIEN), localizada en el sur del estado de Chiapas, México, y es considerada como un área de gran interés para su conservación debido a la gran diversidad biológica que alberga y por sus elevados niveles de productividad primaria estimada (Gutiérrez-Mendieta, Varona-Cordero, \& Contreras-Espinosa, 2006). La REBIEN es la única ANP en el estado de Chiapas que protege las especies de flora y fauna del sistema de humedales costeros, además, está catalogada como la más importante de la costa del pacífico americano, debido a su extensión, estructura y productividad (Contreras, 1988).

Los humedales, a pesar de ser ecosistemas de gran interés, específicamente los manglares, han sido poco abordados desde una perspectiva de estudios de diversidad y riqueza de la mastofauna. Algunas contribuciones destacadas en este sentido han estimado la riqueza, abundancia y patrones de actividad de mamíferos medianos y grandes (Buenrostro-Silva, Sigüenza-Pérez, \& García-Grajales, 2015; Gordillo-Chávez et al., 2015; Hernández-Pérez, Reyna-Hurtado, Castillo-Vela, SanvicenteLópez, \& Moreira-Ramírez, 2015). No obstante, el caso de la REBIEN es destacable, ya que a pesar de ser considerada como el ANP más importante de la costa del pacífico americano, su mastofauna ha sido poco estudiada; entre las especies que han sido objeto de estudios se encuentra el mono araña (Jiménez-Vázquez, 1999), pecarí de collar (Rivadeneyra \& Naranjo, 2004; Torres-Cristiani, 2010) y el jaguar (Juárez, 2002).

En este sentido, el conocimiento de la mastofauna de la REBIEN radica primordialmente en el inventario (Espinoza, Cruz, Kramsky, \& Sánchez, 2003), siendo evidente la ausencia de información sobre estudios ecológicos para la mastofauna de esta zona de Chiapas. Bajo esta premisa, la presente contribución, recaba y enriquece la información sobre el estado que guarda la diversidad de los mamíferos, para fomentar de esta manera el desarrollo de planes de manejo y conservación en la reserva. Con base en esto, el presente trabajo se enfocó a estimar la diversidad y patrones de actividad de los mamíferos medianos y grandes mediante fototrampeo en esta área.

\section{MATERIALES Y MÉTODOS}

Área de estudio: La REBIEN, posee una superficie de 144868 ha en el sur del estado de Chiapas, México $\left(14^{\circ} 43^{\prime}-15^{\circ} 40^{\prime} \mathrm{N} \& 92^{\circ}\right.$ $\left.26^{\prime}-93^{\circ} 20^{\prime} \mathrm{W}\right)$ y tiene una altitud de 0-10 msnm (Tovilla-Hernández, 2007). El clima predominante en esta zona es cálido húmedo Am (w), con una temperatura media anual de $28{ }^{\circ} \mathrm{C}$ y una precipitación anual entre los 2500 y 3000 mm (García, 1973; INE, 1999). La vegetación se compone principalmente de manglares, selvas medianas subperennifolias, selvas bajas caducifolias, zapotonales y tulares, siendo los manglares la vegetación dominante (Tovilla-Hernández, 2007; Romero-Berny \& Tovilla-Hernández, 2009). 
Diseño de muestreo: El estudio se realizó entre agosto 2015 y agosto 2016 mediante el método de fototrampeo abarcando dos temporadas, lluvias (mayo-octubre) y secas (noviembre-abril). Se colocaron 30 estaciones con una trampa-cámara digital (2 LTL Acorn 6210, 8 ScoutGuard SG 550 y 20 Cuddeback C1) en sitios donde se detectaron rastros (p. ej. huellas, excretas, senderos), con una separación de 1 a 3 $\mathrm{km}$ una de otra. Cada estación fue georreferenciada con un geoposicionador (Garmin eTrex 20x). Las trampas-cámara permanecieron activas las 24 horas del día y fueron programadas para sacar una fotografía en intervalos de 60 segundos. Se revisaron cada 30 a 40 días para asegurar su correcto funcionamiento. Las especies fotografiadas fueron identificadas con base en literatura especializada (Ceballos \& Oliva, 2005; Aranda, 2012). Para la clasificación y nomenclatura utilizadas se siguió el trabajo de Ceballos \& Arroyo-Cabrales (2012). En relación con el tamaño corporal de los mamíferos y basándonos en los trabajos de Arita \& Figueroa (1999) y Ceballos \& Oliva (2005), se consideraron los siguientes criterios para definir las tallas de mamíferos medianos y grandes: medianos $>101 \mathrm{~g} \mathrm{y}<10 \mathrm{~kg}$; grandes $>10 \mathrm{~kg}$.

Riqueza de especies: La riqueza se determinó como el número de especies por temporada de muestreo. Para esto se utilizó un análisis de interpolación y extrapolación propuesto por Chao y colaboradores (2014), utilizando los números de Hill (Hill, 1973) para la riqueza de especies $(\mathrm{q}=0)$ con base en datos de incidencia. Así también se estimó la riqueza por cobertura de muestreo, que es una medida de la completitud de la muestra con respecto al tamaño de la comunidad, así una comunidad bien muestreada debería tener una cobertura de $100 \%$ (Chao \& Jost, 2012). El análisis se realizó con el paquete iNEXT (Hsieh, Ma, \& Chao, 2016), integrado al programa estadístico R (v3.4.0) (R CoreTeam, 2017).

Abundancia de especies: Debido a que no fue posible identificar a nivel individual los registros de todas las especies, se consideraron como independientes cuando se obtuvieron fotografías consecutivas de individuos claramente diferenciables o las fotografías consecutivas de individuos de la misma especie que estaban separadas por más de 24 horas entre un evento y el otro (Srbek-Araujo \& Chiarello, 2005; Chávez et al., 2013). En especies con hábitos gregarios, el número de registros independientes considerado fue igual al número de individuos observados en la misma (Maffei, Cuellar, \& Noss, 2002). Para obtener el índice de abundancia relativa (IAR) de cada especie se utilizó la siguiente ecuación: $\mathrm{IAR}=(\mathrm{C} /$ $\mathrm{EM}) * 100$ días/trampa. Donde: $\mathrm{C}=$ número de eventos fotografiados; EM = esfuerzo de muestreo (número de cámaras-trampa utilizadas por días de monitoreo) y 100 días trampa (factor de corrección estándar) (Lira-Torres, BrionesSalas, \& Sánchez-Rojas, 2014). Se compararon los valores del IAR para todo el conjunto de especies entre las dos temporadas y para cada una de las especies por temporada a través de la prueba de "U" de Mann-Whitney, considerando un nivel de significancia $\mathrm{P}<0.05$ (Sokal \& Rohlf, 1981).

Patrón de actividad: Se determinó el patrón de actividad de aquellas especies más abundantes. Los registros obtenidos de cada especie se ordenaron por intervalos de una hora y la actividad se agrupó en cuatro periodos: a) diurno (08:01-17:59); b) nocturno 20:01-05:59), c) crepuscular (06:00-08:00 y 18:00-20:00) y d) catemeral (especies que no mostraron un patrón claro de actividad) (Monroy-Vilchis, Zarco-González, Rodríguez-Soto, Soria-Díaz, \& Urios, 2011). El periodo crepuscular se delimitó una hora antes y una hora después del amanecer y atardecer utilizando el programa SUN TIMES v7.1 (Kay \& Du Croz, 2008). Para describir los patrones de actividad de las especies se elaboraron histogramas circulares. Asimismo, se realizaron pruebas de Rayleigh para evaluar la distribución de los registros de cada una de las especies durante las $24 \mathrm{hr}$ del día y se compararon los patrones de actividad entre pares de especies a través de la prueba estadística $\mathrm{U}^{2}$ de Watson (Zar 1998), 
utilizando un nivel de significancia $\mathrm{P}<0.05$. Los histogramas y los análisis estadísticos se realizaron mediante el software Oriana Versión 4.0 (Kovach Computing Services, 2011).

\section{RESULTADOS}

Riqueza de especies: Se obtuvo un total de 1851 registros independientes de 5554 fotografías, empleando un esfuerzo de muestreo de 5400 días-trampa. Se registraron 19 especies de mamíferos medianos y grandes, pertenecientes a 15 familias y 7 órdenes. Carnívora fue el orden mejor representado, con 5 familias y 9 especies registradas (Cuadro 1). La riqueza de especies de mamíferos $(\mathrm{q}=0)$ fue ligeramente mayor en la temporada seca $(\mathrm{N}=18)$ que en la de lluvias $(\mathrm{N}=16)$. Se destaca una tendencia asintótica en las curvas de acumulación de especies para ambas temporadas (Fig. 1). La cobertura (completitud) de la muestra para la temporada seca y de lluvias fue de $99.9 \%$ y $99.8 \%$ respectivamente, indicando que el muestreo fue representativo en ambos casos. De las especies registradas, seis se consideran bajo algún estado de riesgo dentro de la legislación mexicana (SEMARNAT, 2010): el jaguar Panthera onca y el oso hormiguero Tamandua mexicana en peligro de extinción; jaguarundi Puma yagouaroundi, grisón Galictis vittata, nutria neotropical Lontra longicaudis y puerco espín tropical Sphiggurus mexicanus, como amenazadas.

Abundancia relativa: Las especies con mayor abundancia relativa en ambas temporadas fueron el mapache Procyon lotor (IAR = $4.35, \mathrm{~N}=493)$ y el coatí Nasua narica (IAR = 3.91, $\mathrm{N}=444)$, seguidas del tlacuache cuatro ojos Philander opossum (IAR $=2.04, \mathrm{~N}=232$ ), el tepezcuintle Cuniculus paca (IAR $=1.89$, $\mathrm{N}=215$ ), el tlacuache Didelphis marsupialis (IAR $=1.67, \mathrm{~N}=190)$ y el armadillo Dasypus novemcinctus (IAR $=1.02, \mathrm{~N}=116$ ) (Cuadro 1). En cuanto a las abundancias relativas entre la temporada de lluvias y secas, no existieron diferencias estadísticas significativas $(\mathrm{Z}=$ $-0.26, \mathrm{P}=0.80)$. El análisis de cada una de las especies por temporada muestra que la abundancia estimada para el puerco espín tropical

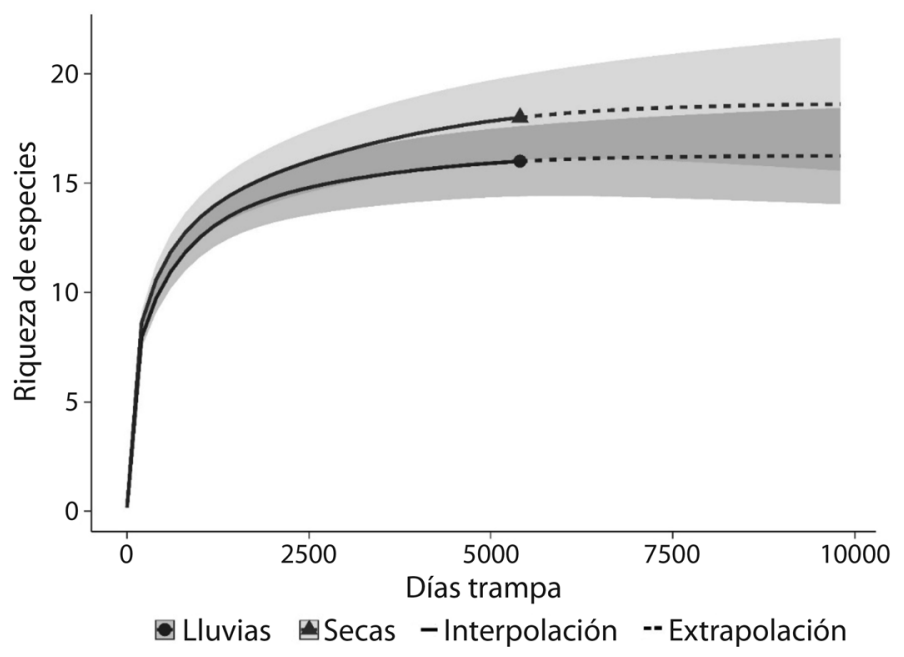

Fig. 1. Curvas de interpolación y extrapolación basadas en el esfuerzo de muestreo (días-trampa) para la riqueza de especies de mamíferos $(q=0)$. Las muestras de referencia se señalan con símbolos (círculo: lluvias; triángulo: secas). La zona sombreada señala los intervalos de confianza al $95 \%$. La extrapolación se extiende hasta el doble del esfuerzo de muestreo de referencia.

Fig. 1. Interpolation and extrapolation curves based on sampling effort (trap-days) for the mammalian species richness $(q=0)$. The reference samples are marked with symbols (circle: rainy; triangle: dry). The shaded area indicates the $95 \%$ confidence intervals. The extrapolation extends up to twice the reference sampling effort. 
CUADRO 1

Mamíferos medianos y grandes registrados en la Reserva de la Biosfera La Encrucijada, Chiapas, México

TABLE 1

Medium and large mammals recorded in La Encrucijada Biosphere Reserve, Chiapas, México

\begin{tabular}{|c|c|c|c|c|c|c|c|}
\hline \multirow{2}{*}{ ORDEN/Familia/Especie } & \multicolumn{2}{|c|}{ Secas } & \multicolumn{2}{|c|}{ Lluvias } & \multicolumn{2}{|c|}{ Total } & \multirow{2}{*}{ NOM-059 } \\
\hline & N Reg & IAR & N Reg & IAR & N Reg & IAR & \\
\hline \multicolumn{8}{|l|}{ CARNIVORA } \\
\hline \multicolumn{8}{|l|}{ Procyonidae } \\
\hline Procyon lotor & 233 & 4.13 & 260 & 4.56 & 493 & 4.35 & \\
\hline Nasua narica & 191 & 3.39 & 253 & 4.44 & 444 & 3.91 & \\
\hline \multicolumn{8}{|l|}{ Felidae } \\
\hline Puma yagouaroundi & 14 & 0.25 & 16 & 0.28 & 30 & 0.26 & A \\
\hline Panthera onca & 5 & 0.09 & 2 & 0.04 & 7 & 0.06 & $\mathrm{P}$ \\
\hline \multicolumn{8}{|l|}{ Canidae } \\
\hline Urocyon cinereoargenteus & 16 & 0.28 & 6 & 0.11 & 22 & 0.19 & \\
\hline Canis latrans & 12 & 0.21 & 8 & 0.14 & 20 & 0.18 & \\
\hline \multicolumn{8}{|l|}{ Mephitinae } \\
\hline Conepatus leuconotus & 2 & 0.04 & 10 & 0.18 & 12 & 0.11 & \\
\hline \multicolumn{8}{|l|}{ Mustelidae } \\
\hline Galictis vittata & 0 & 0 & 2 & 0.04 & 2 & 0.02 & A \\
\hline Lontra longicaudis & 1 & 0.02 & 0 & 0 & 1 & 0.01 & A \\
\hline \multicolumn{8}{|l|}{ DIDELPHIMORPHIA } \\
\hline \multicolumn{8}{|l|}{ Didelphidae } \\
\hline Philander opossum & 96 & 1.7 & 136 & 2.38 & 232 & 2.04 & \\
\hline Didelphis marsupialis & 97 & 1.72 & 93 & 1.63 & 190 & 1.67 & \\
\hline \multicolumn{8}{|l|}{ RODENTIA } \\
\hline \multicolumn{8}{|l|}{ Cuniculidae } \\
\hline Cuniculus paca & 90 & 1.6 & 125 & 2.19 & 215 & 1.89 & \\
\hline \multicolumn{8}{|l|}{ Dasyproctidae } \\
\hline Dasyprocta punctata & 2 & 0.04 & 7 & 0.12 & 9 & 0.08 & \\
\hline \multicolumn{8}{|l|}{ Erethizontidae } \\
\hline Sphiggurus mexicanus & 7 & 0.12 & 0 & 0 & 7 & 0.06 & A \\
\hline \multicolumn{8}{|l|}{ CINGULATA } \\
\hline \multicolumn{8}{|l|}{ Dasypodinae } \\
\hline Dasypus novemcinctus & 72 & 1.28 & 44 & 0.77 & 116 & 1.02 & \\
\hline \multicolumn{8}{|l|}{ ARTIODACTYLA } \\
\hline \multicolumn{8}{|l|}{ Tayassuidae } \\
\hline Pecari tajacu & 21 & 0.37 & 12 & 0.21 & 33 & 0.29 & \\
\hline \multicolumn{8}{|l|}{ Cervidae } \\
\hline Odocoileus virginianus & 2 & 0.04 & 1 & 0.02 & 3 & 0.03 & \\
\hline \multicolumn{8}{|l|}{ PILOSA } \\
\hline \multicolumn{8}{|l|}{ Myrmecophagidae } \\
\hline Tamandua mexicana & 9 & 0.16 & 5 & 0.09 & 14 & 0.12 & $\mathrm{P}$ \\
\hline LAGOMORPHA & & & & & & & \\
\hline Leporidae & & & & & & & \\
\hline Sylvilagus sp. & 1 & 0.02 & 0 & 0 & 1 & 0.01 & \\
\hline
\end{tabular}

$\mathrm{N}$ reg = Número de registros independientes; IAR: Índice de abundancia relativa; NOM-059 NOM-059-SEMARNAT: A = Amenazada, $\mathrm{P}=$ En peligro de extinción.

$\mathrm{N}$ reg $=$ Number of independent records; RAI = Relative abundance index; NOM-059-SEMARNAT-2010: T = Threatened, $\mathrm{E}=$ Endangered. 
es mayor en la temporada seca $(\mathrm{Z}=-3.31$, $\mathrm{P}$ $=0.002$ ). Para el resto de las especies no hubo diferencias significativas en las estimaciones de abundancia entre temporadas.

Actividad: La distribución de la actividad de las seis especies de mamíferos más abundantes fue heterogénea a lo largo del día, con base a los resultados de la prueba de Rayleigh $(\mathrm{P}<0.001)$. Procyon lotor presentó un pico de actividad entre las 20:00 y 06:00 hr (media $=01: 04 \mathrm{~h} \pm 37 \mathrm{~min}$ ), aunque mostró un comportamiento mayormente nocturno $(66.9 \%)$ se considera catemeral, ya que se encontró activo tanto en el día como en horas crepusculares ( $15.7 \%$ y $17.3 \%$ de sus registros, respectivamente). El $78.2 \%$ de los registros de Nasua narica fueron diurnos, con un pico de actividad entre las 10:00 y 12:00 hr (media $=12: 20 \mathrm{~h}$ $\pm 56 \mathrm{~min})$. Cuatro especies fueron nocturnas, Philander opossum $(88.7 \%)$, con un pico de actividad entre las 20:00 y 22:00 hr (media $=$ 00:24 h \pm 01:06 min); Cuniculus paca (94.4 $\%$ ), con un pico de actividad entre las 20:00 y 22:00 hr (media $=23: 39 \mathrm{hr} \pm 01: 13 \mathrm{~h})$; Didelphis marsupialis $(79.8 \%$ ), con un pico de actividad entre las 20:00 y 22:00 hr (media $=23: 17$ $\mathrm{hr} \pm$ 01:04 hr) y Dasypus novemcinctus (84.21 $\%)$, con un pico de actividad entre las 20:00 y 04:00 hr (media $=23: 58 \mathrm{hr} \pm$ 01:01 hr) (Fig. 2). De acuerdo con la prueba de $\mathrm{U}^{2}$ de Watson, encontramos estadísticamente patrones de actividad similares entre $D$. novemcinctus con tres especies; P. opossum $\left(\mathrm{U}^{2}=0.08, \mathrm{P}>0.2\right), C$. paca $\left(\mathrm{U}^{2}=0.15, \mathrm{P}>0.05\right)$ y D. marsupialis $\left(\mathrm{U}^{2}\right.$ $=0.18, \mathrm{P}>0.05)$.

\section{DISCUSION}

Este es el primer estudio que estima la riqueza y abundancia de las especies de mamíferos de talla mediana o grande de la REBIEN. Se registró el $38 \%$ de los mamíferos de esa categoría registrados para el estado (49 especies; Rivero \& Medellín, 2015). La riqueza de 19 especies obtenida en este estudio fue mayor a la registrada en otras zonas tropicales con vegetación similar, donde los valores van de
11 a 15 especies (CONANP, 2011b; CONANP, 2011c; Buenrostro-Silva et al., 2015). Dado el intensivo esfuerzo de muestreo (considerando los análisis de curvas de interpolación y extrapolación) este puede ser considerado como una muy buena aproximación a la riqueza y diversidad de especies de mamíferos medianos y grandes para el área.

Se confirma la presencia de seis especies de mamíferos considerados como amenazados y en peligro de extinción para México (SEMARNAT, 2010), resaltando la importancia de la REBIEN en la conservación de especies de este grupo. Sin embargo, nuestras observaciones indican que el área tiene presiones de cacería, ya que se obtuvieron varios registros de cazadores y robo de equipo por esta causa. Por otro lado, algunas de las áreas de la REBIEN no fueron muestreadas, debido a que son inaccesibles en temporada de lluvias o inseguras debido a la presencia de cazadores o al robo de cámaras, por lo que un diseño de muestreo espacialmente más extenso, sobre todo en aquellas áreas que no se pudieron acceder, probablemente aumentaría el número de especies y proporcionaría más información sobre su distribución y el uso del hábitat en el área.

Los análisis de interpolación y extrapolación indicaron una diferencia en la riqueza, aunque no significativa, de especies entre temporadas, donde fue ligeramente mayor en la temporada seca, registrando dos especies más que en la temporada de lluvias. Esto es posiblemente debido a que algunas especies se ven forzadas a aumentar el área de forrajeo, debido a la escasez de alimentos en esta temporada, lo que incrementa la probabilidad de que sean registrados (Holzman, Conroy, \& Pickering, 1992; Valenzuela \& Macdonald, 2002).

Se confirma la presencia de 19 especies de mamíferos para la REBIEN, 14 de ellas previamente registradas (e.g. Espinoza et al., 2003), excepto Puma yagouaroundi, Didelphis marsupialis, Sylvilagus sp, Canis latrans y Conepatus leuconotus. Las especies más abundantes de acuerdo con el IAR fueron Procyon lotor $(\mathrm{IAR}=4.35)$, seguida de Nasua narica 


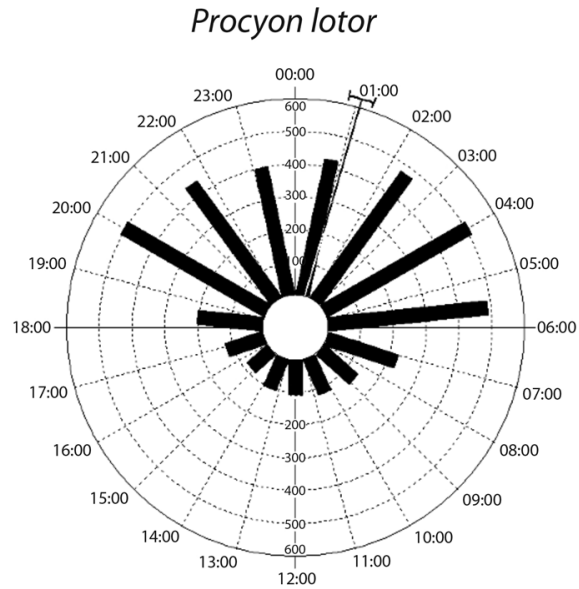

Philander opossum

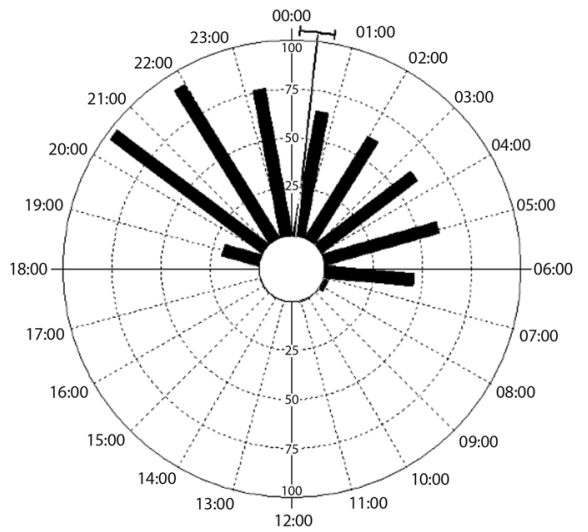

Dasypus novemcinctus

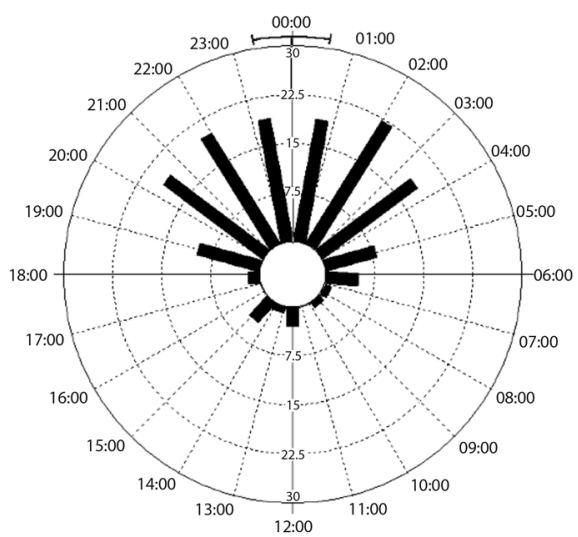

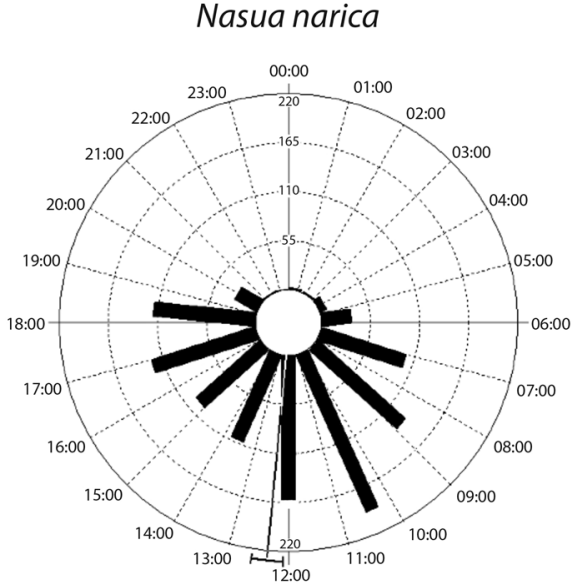

Didelphis marsupialis

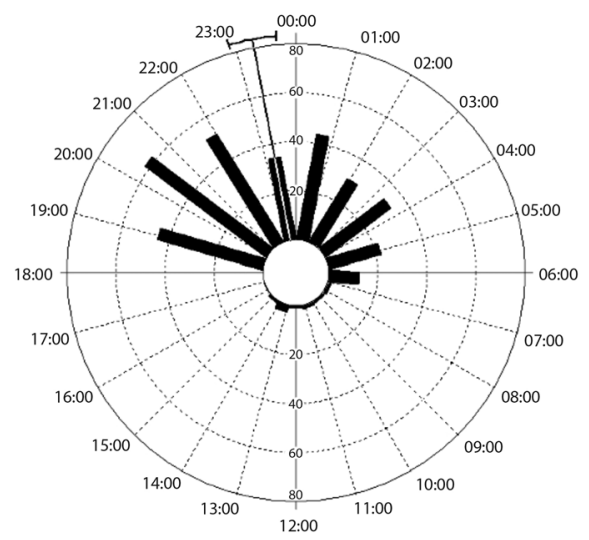

Cuniculus paca

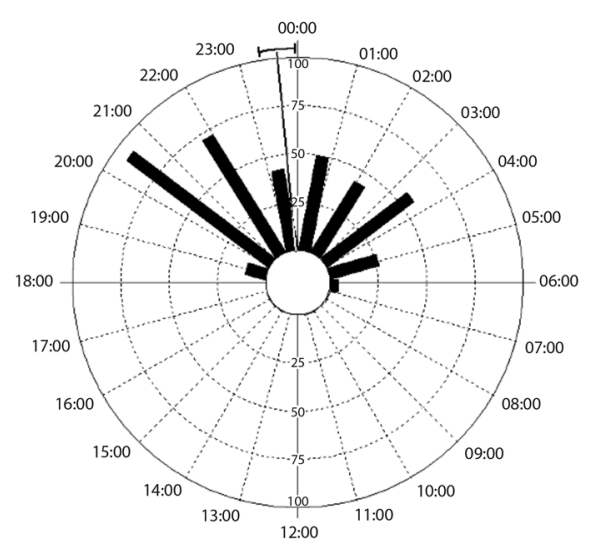

Fig. 2. Patrones de actividad de las especies más abundantes en la REBIEN. La longitud de las barras indica la cantidad de imágenes registradas. La línea recta indica el valor de la media y el arco perpendicular muestra la amplitud del intervalo de confianza del $95 \%$.

Fig. 2. Activity patterns of most abundant species in ENBIRE. The length of bars indicates the number of images recorded. The straight line indicates the mean value and the perpendicular arc shows the amplitude of the $95 \%$ confidence interval. 
$(\mathrm{IAR}=3.91)$. Estos resultados coinciden con los obtenidos en la Reserva de la Biosfera Marismas Nacionales (RBMN) (CONANP, 2011c), donde Procyon lotor fue la especie más abundante (IAR $=1.86)$. Sin embargo, para otros sitios los registros se encuentran muy por debajo en relación con este estudio, tal es el caso de la selva baja caducifolia en la Planicie Costera de Tehuantepec (IAR $=0.01$ ) (CortésMarcial \& Briones-Salas, 2014). En el caso de Nasua narica, nuestros resultados coinciden con lo reportado en una zona de petenes de la costa de la Península de Yucatán, donde esta especie fue la más abundante $(\mathrm{IAR}=222.51)$ (Hernández-Pérez et al., 2015); asimismo, para la región de los Chimalapas fue de las especies con mayor abundancia (IAR $=4.11$ ) (LiraTorres \& Briones-Salas, 2012).

En general, para Procyon lotor y Nasua narica es común que se registren valores altos de abundancia, ya que son especies generalistas u oportunistas y que poseen una capacidad de adaptación para sobrevivir y desarrollarse en una gran variedad de ambientes, aún con la presencia de actividad humana (Valenzuela \& Ceballos, 2000; Pérez-Irineo \& SantosMoreno, 2010).

En la REBIEN, se destaca la presencia de dos especies de felinos, Puma yagouaroundi y Panthera onca, ambos con un IAR bajo (0.25 y 0.09 respectivamente). Estos resultados contrastan en parte con lo encontrado para la RBMN (CONANP, 2011c), donde $P$. onca fue la especie más abundante (IAR $=2.65,4$ hembras y 5 machos) contrario a $P$. yagouaroundi, que fue la menos abundante (IAR = 0.06). Por su parte, para el Área de Protección de Flora y Fauna Laguna de Términos, se reportaron 8 individuos de $P$. onca (HidalgoMihart, Contreras-Moreno, \& Pérez-Solano, 2012). En el presente estudio se obtuvo el registro de un individuo (hembra), aunque se esperaban obtener más registros de $P$. onca, dado que existen grandes extensiones de áreas con vegetación natural y de una gran abundancia de especies de presas como Procyon lotor, Nasua narica, Cuniculus paca y Dasypus novemcinctus (Ávila-Nájera, Rosas-Rosas,
Tarango-Arámbula, Martínez-Montoya, \& Santoyo-Brito, 2011). Sin embargo, Aranda (1996) y Cruz, Palacios, \& Güiris (2007), mencionan que la REBIEN es una reserva aislada y debido a su forma alargada puede llegar a fraccionarse con facilidad, por lo que la persistencia a largo plazo de los jaguares en la reserva dependerá de las acciones que se desarrollen para conectar la Reserva con otras áreas naturales.

Dentro de las especies que se registraron, el grisón $G$. vittata es una de las cuales se desconoce su estado real de conservación, ya que no existe información sobre la biología y ecología, ni datos sobre la abundancia de sus poblaciones en México (De la Torre, Muench, $\&$ Arteaga, 2009), es considerada una especie rara; el conejo Sylvilagus sp., es una especie con una amplia distribución geográfica, sin embargo, no existía referencia de que sugiriera su presencia dentro de la REBIEN, hasta que se obtuvo la fotografía de un individuo. Estudios en México sobre la abundancia de la nutría neotropical $L$. longicaudis han aumentado en los últimos años (Casariego-Madorell, List, \& Ceballos 2008; Cruz-García, Contreras-Balderas, Nava-Castillo, \& Gallo-Reynoso, 2017). Esta especie es sensible a la degradación de los ríos y a la contaminación de los cuerpos de agua, por lo que su presencia permite inferir que la calidad del agua en la REBIEN es aceptable (Kasper, Bastazini, Salvi, \& Grillo, 2008; Monroy-Vilchis \& Mundo, 2009).

En el caso de las dos especies de cánidos registrados, Canis latrans y Urocyon cinereoargenteus (IAR $=0.28$ y 0.29 respectivamente), se esperaba obtener una mayor abundancia de ambas, dado que son especies muy generalistas y se les considera comunes en la región. Sin embargo, se ha registrado que $C$. latrans es menos abundante en zonas boscosas y tropicales (Servín \& Chacón, 2005).

En relación con los ungulados, el IAR de Pecari tajacu $(\mathrm{IAR}=0.37)$ fue relativamente bajo a comparación de otro estudio en ambientes semejantes (Hernández-Pérez et al., 2015). Anteriormente, esta especie fue extirpada en la REBIEN, debido a esto se realizó un 
programa de reintroducción en la reserva por parte de autoridades ambientales de México en el año 2006, con la intención de reestablecer poblaciones genética y ecológicamente viables en esta zona (CONANP, 2006a). Con relación a Odocoileus virginianus, sus abundancias son bajas $(\mathrm{IAR}=0.04)$ en comparación a otros estudios (Faller-Menéndez, Urquiza-Haas, Chávez, Johnson, \& Ceballos 2005; CONANP, 2011b), no registrándose en el manglar, selva mediana y selva baja. Los únicos registros obtenidos fueron cerca de zonas cultivadas y con presencia de ganado, donde se sabe que las poblaciones de venado cola blanca son muy bajas en ese tipo de sitios (Contreras-Moreno, Zúñiga-Sánchez, \& Bello-Gutiérrez, 2015).

En cuanto a la abundancia relativa de mamíferos de talla mediana como Philander opossum, Didelphis marsupialis, Cuniculus paca y Dasypus novemcinctus, fue mayor en relación con otras especies que componen la comunidad de mamíferos medianos y grandes de la región. Otras especies registradas, aunque poco abundantes fueron el oso hormiguero Tamandua mexicana y el puerco espín tropical Sphigurus mexicanus, lo cual puede estar relacionado con el diseño experimental utilizado, ya que está orientado a individuos de hábitos terrestres, por lo que las especies con hábitos arborícolas difícilmente se pueden detectar (Aranda, 2012).

El tamaño corporal de los mamíferos está relacionado con su patrón de actividad, de manera que los grandes mamíferos del neotrópico que tienen requerimientos energéticos mayores forrajean durante todo el día (LiraTorres \& Briones-Salas, 2012), y los pequeños mamíferos tienden a ser nocturnos como una estrategia antidepredación (Van Schaik \& Griffiths, 1996). Monroy-Vilchis et al., (2011) mencionan que el patrón de actividad de especies de hábitos nocturnos/crepusculares con peso $<10 \mathrm{~kg}$ (Procyon lotor, Nasua narica, Didelphis marsupialis, Philander oposum y Cuniculus paca) se relaciona con la evasión del riesgo de depredación. Nuestros resultados contradicen esa afirmación, ya que $N$. narica y $P$. lotor mostraron actividad durante el día, por lo que es probable que factores como la disponibilidad de alimento o presas, o algún otro eje del nicho ecológico tengan mayor influencia sobre su patrón de actividad (Albanesi, Jayat, \& Brown, 2016). Existen trabajos que reportan a $N$. narica como diurno (ej. Valenzuela \& Ceballos, 2000) y $P$. lotor de actividad catemeral (ej. González-Maya, Schipper, \& Benítez 2009). Sin embargo, otras especies de mamíferos como Philander opossum, Cuniculus paca, Dasypus novemcinctus y Didelphis marsupialis concuerdan con lo reportado por Van Schaik y Griffiths (1996), y otros estudios (Lira-Torres \& Briones-Salas, 2012; Hernández-Pérez et al., 2015), mostrándose activos durante la noche.

A pesar de que la REBIEN mantiene una mayor parte de la vegetación original, algunos sitios están perturbados por cambios en el uso del suelo destinados principalmente a la agricultura y ganadería, incendios forestales ligados directamente con el saqueo de fauna y la cacería, sin embargo, se desconoce el impacto real que pueden tener estas actividades. Aunque la reserva presenta diferentes niveles de intervención humana, brinda las condiciones apropiadas y satisface los requerimientos básicos para la persistencia de algunas especies de mamíferos medianos y grandes.

Es importante mencionar que especies como el jaguar, jaguarundi y la nutria neotropical son especies prioritarias para la conservación debido a su importancia como especies sombrilla, por lo tanto, para conservarlas se debe de incluir a las redes tróficas de la sque dependen en la REBIEN, se debe contemplar el manejo integral de sus poblaciones y del hábitat a lo largo de su espacio geográfico.

A su vez se deben de impulsar alternativas viables de manejo integrado con las comunidades rurales que reduzcan el impacto de las actividades humanas sobre las especies y su hábitat, procurando generar un bienestar económico en las poblaciones rurales, como se ha realizado en otros ambientes similares (Domínguez-Domínguez, Zavala-Cruz, \& Martínez-Zurimendi, 2011; Sanjurjo-Rivera \& Campos-Palacín, 2011). Asimismo, se debe enfatizar la importancia de la creación de 
comités de vigilancia comunitaria especializada en fauna silvestre y la necesidad de reforzarlos con equipo y capacitación para un mejor desempeño, ya que ellos juan un papel muy importante en la detección de prácticas ilícitas y en la conservación de la flora y fauna de la REBIEN. Para que los planes de manejo puedan cumplir su papel de guiar la conservación dentro de las áreas naturales protegidas, deben estar fundamentados en información confiable y de calidad, en este sentido, nuestro trabajo es una contribución esencial a la línea base para el manejo de la REBIEN.

\section{RESUMEN}

En México, los humedales ocupan una porción importante del país, entre ellos, los manglares son particularmente diversos. La Reserva de la Biosfera de La Encrucijada (REBIEN) es un ecosistema costero de manglares localizado en la planicie costera del pacífico en la porción suroeste del estado de Chiapas. Los manglares de la REBIEN se encuentran entre los más importantes de la costa pacífica americana, destacando el papel que juegan en el mantenimiento de la comunidad de mamíferos en la costa del estado. Existen pocos estudios en México relacionados con los mamíferos asociados a estos ecosistemas, nuestro objetivo fue estimar la diversidad y los patrones de actividad de los mamíferos medianos y grandes en la REBIEN. El muestreo se realizó de agosto de 2015 a agosto de 2016 utilizando trampas-cámara. Se obtuvieron un total de 1851 registros de fotografías independientes con un esfuerzo de muestreo total de 5400 días-trampa. Se registraron 19 especies de mamíferos pertenecientes a 15 familias y siete órdenes, y cinco especies fueron nuevos registros en el área. Seis especies presentes figuran en la lista, dos en peligro de extinción y cuatro amenazadas. Los análisis de interpolación y extrapolación mostraron una tendencia asintótica en las curvas para las estaciones de lluvia y seca. La cobertura de la muestra para ambas temporadas fue de $99.9 \%$ y $99.8 \%$, respectivamente, lo que indica en ambos casos un muestreo representativo. Según el índice de abundancia relativa obtenido, las especies más abundantes fueron Procyon lotor (IAR $=4.35$ ), Nasua narica $(\mathrm{IAR}=3.91)$, Philander opossum $(\mathrm{IAR}=$ 2.04), Cuniculus paca (IAR = 1.89), Didelphis marsupialis $(\mathrm{IAR}=1.67)$ y Dasypus novemcinctus $(\mathrm{IAR}=1.02)$. En términos de patrones de actividad, C. paca, P. opossum, $D$. marsupialis y $D$. novemcinctus fueron principalmente nocturnos; $P$. lotor mostró una tendencia hacia hábitos nocturnos, pero también se registró durante el día; $N$. narica fue principalmente diurno. Esta información puede ser útil para la creación de programas de manejo y la conservación de mamíferos medianos y grandes en la REBIEN, especialmente para especies en riesgo.

Palabras clave: trampas-cámara; riqueza de especies; humedales; ecología de mamíferos silvestres.

\section{REFERENCIAS}

Albanesi, S. A., Jayat, J. P., \& Brown, A. D. (2016). Patrones de actividad de medio y gran aporte en el pedemonte de yungas del noreste argentino. Mastozoología Neotropical, 23(2), 335-358.

Aranda, M. (1996). Distribución y abundancia del jaguar, Panthera onca (Carnivora: Felidae) en el estado de Chiapas, Mexico. Acta Zoológica Mexicana (n.s), $68,45-52$.

Aranda, M. (2012). Manual para el rastreo de mamiferos silvestres de México. México: CONABIO.

Arita, H. \& Figueroa, F. (1999). Geographic patterns of body-mass diversity of Mexican mammals. Oikos, 85, 310-319.

Ávila-Nájera, D. M., Rosas-Rosas, O. C., TarangoArámbula, L. A., Martínez-Montoya, J. F., \& Santoyo-Brito, E. (2011). Conocimiento, uso y valor cultural de seis presas del jaguar (Panthera onca) y su relación con éste, en San Nicolás de los Montes, San Luis Potosí, Mexico. Revista Mexicana de Biodiversidad, 82, 1020-1028.

Buenrostro-Silva, A., Sigüenza-Pérez, D., \& García-Grajales, J. (2015). Mamíferos carnívoros del Parque Nacional Lagunas de Chacahua: Riqueza, abundancia y patrones de actividad. Revista Mexicana de Mastozoología Nueva época, 5(2), 39-54.

Casariego-Madorell, M. A., List, R., \& Ceballos, G. 2008. Tamaño poblacional y alimentación de la nutria de río (Lontra longicaudis annectens) en la costa de Oaxaca, México. Acta Zoológica Mexicana (n.s.), 24, 179-200.

Ceballos, G., \& Arroyo Cabrales, J. (2012). Lista actualizada de los mamíferos de México 2012. Revista Mexicana de Mastozoología Nueva época, 2(1), 27-80.

Ceballos, G., \& Oliva, G. (2005). Los mamiferos silvestres de México. México: CONABIO-Fondo de Cultura Económica.

Chao, A., Gotelli, N. J., Hsieh, T. C., Sander, E. L., Ma, K. H., Colwell, R. K., \& Ellison, A.M. (2014). Rarefaction and extrapolation with Hill numbers: a framework for sampling and estimation in species diversity studies. Ecological Monographs, 84, 45-67.

Chao, A., \& Jost, L. (2012). Coverage-based rarefaction: standardizing samples by completeness rather than by size. Ecology, 93(12), 2533-2547. 
Chávez, C., De la Torre, A., Bárcenas, H., Medellín, R. A., Zarza, H., \& Ceballos, G. (2013). Manual de fototrampeo para estudio de fauna silvestre: el jaguar en México como estudio de caso. México: Alianza WWF, Telcel, Universidad Nacional Autónoma de México.

CONANP (2006a). Reintroducción experimental de pecarí de collar (Tayassu tajacu) para repoblar zonas de distribución histórica en la Reserva de la Biosfera La Encrucijada: Un esfuerzo conjunto para la conservación de la vida silvestre. Documento interno. Propuesta de investigación.

CONANP. (2011b). Monitoreo de jaguar (Panthera onca) en el Corredor Laguna de Términos-Calakmul, con énfasis en la Región Prioritaria para la Conservación Chenkan. México: CONANP. Recuperado de: https://simec.conanp.gob.mx/pdf_monitoreo/118APFF\%20Laguna\%20de\%20Terminos_Jaguar.pdf

CONANP. (2011c). Monitoreo de Jaguar (Panthera onca) en la Reserva de la Biosfera Marismas Nacionales. México: CONANP. Recuperado de: http://www. conanp.gob.mx/acciones/fichas/marismas_nacionales/info.pdf

Contreras, F. (1988). Las Lagunas Costeras Mexicanas. México, D. F.: Centro de Ecodesarrollo, SEPESCA.

Contreras-Moreno, F. M., Zúñiga-Sánchez, J. S., \& BelloGutiérrez, J. (2015). Parámetros poblacionales de Odocoileus virginianus (Cervidae) en dos comunidades de Tabasco, México. Revista Latinoamericana de Conservación, 4, 7-13.

Cortés-Marcial, M., \& Briones-Salas, M. (2014). Diversidad, abundancia relativa y patrones de actividad de mamíferos medianos y grandes en una selva seca del Istmo de Tehuantepec, Oaxaca, México. Revista de Biología Tropical, 62(4), 1433-1448.

Cruz-García, F., Contreras-Balderas, A. J., Nava-Castillo, R., \& Gallo-Reynoso, J. P. (2017). Habitat and abundance of the Neotropical otter (Lontra longicaudis annectens) in Pueblo Nuevo, Durango, México. Therya, 8(2), 123-130.

Cruz, E., Palacios, G., \& Güiris, M. (2007). Situación actual del jaguar en Chiapas. In G. Ceballos., C. Chávez., R. List., \& H. Zarza (Eds.), Conservación y manejo del jaguar en México: estudios de caso y perspectivas (pp. 81-89). México: CONABIO, Alianza WWF, Telcel, Universidad Nacional Autónoma de México.

De la Torre, A., Muench, C., \& Arteaga, M. C. (2009). Nuevos registros de grisón Galictis vittata, para la selva Lacandona, Chiapas, México. Revista Mexicana de Mastozoología, 13, 109-114.

Domínguez-Domínguez, M., Zavala-Cruz, J., \& MartínezZurimendi, P. (2011). Manejo forestal sustentable de los manglares de Tabasco. México: Secretaría de
Recursos Naturales y Protección Ambiental, Colegio de Postgraduados.

Espinoza, E., Cruz, E., Kramsky, H., \& Sánchez, I. (2003). Mastofauna de la reserva de la biosfera "La Encrucijada”, Chiapas. Revista Mexicana de Mastozoología, 7, 5-19.

Faller-Menéndez, J. C., Urquiza-Haas, T., Chávez, C., Johnson, S., \& Ceballos, G. (2005). Registros de mamíferos en la reserva privada el zapotal, en el noreste de la península de Yucatán. Revista Mexicana de Mastozoología, 9, 128-140.

García, E. (1973). Modificación al sistema de clasificación climática de Köppen. México: Instituto de Geografía, UNAM.

González-Maya, J. F., Schipper, J., \& Benítez, A. (2009). Activity patterns and community ecology of small carnivores in the Talamanca region, Costa Rica. Small Carnivore Conservation, 41, 9-14.

Gordillo-Chávez, E. J., Mata Zayas, E., García-Morales, R., Morales-Garduza, M.A., Villanueva-García, C., \& Valdez-Lea, J. D. (2015). Mastofauna del humedal Chaschoc-Sejá en Tabasco, México. Therya, 6(3), 535-544.

Gutiérrez-Mendieta, F. J., Varona-Cordero, F., \& Contreras-Espinosa, F. (2006). Caracterización estacional de las condiciones físico-químicas y de productividad primaria fitoplanctónica de dos lagunas costeras tropicales del estado de Chiapas, México. Hidrobiológica, 16, 137-146.

Hernández-Pérez, E., Reyna-Hurtado, R., Castillo-Vela, G., Sanvicente-López, M., \& Moreira-Ramírez, J. F. (2015). Fototrampeo de mamíferos terrestres de talla mediana y grande asociados a petenes del noroeste de la península de Yucatán, México. Therya, 6(3), 559-574.

Hidalgo-Mihart, M. G., Contreras-Moreno, F., \& PérezSolano, L. (2012). Jaguares de los humedales del sureste de México. Biodiversitas, 104, 6-11.

Hill, M. O. (1973). Diversity and Evenness: A unifying notation and its consequences. Ecology, 54, 427-432.

Holzman, S., Conroy, M. J., \& Pickering, J. (1992). Home range, movements, and habitat use of coyotes in Southcentral Georgia. Journal of Wildlife Management, 56, 139-146.

Hsieh, T. C., Ma, K. H., \& Chao, A. (2016). iNEXT: an R package for rarefaction and extrapolation of species diversity (Hill numbers). Methods in Ecology and Evolution, 7, 1451-1456.

INE (Instituto Nacional de Ecología). (1999). Programa de Manejo de la Reserva de la Biosfera La Encrucijada. México: Secretaría de Medio Ambiente Recursos Naturales y Pesca. 
Jiménez-Vázquez, L. A. (1999). Distribución y abundancia del mono araña (Ateles geoffroyi vellerosus) en los humedales de la reserva de la biosfera La Encrucijada, Chiapas (Tesis de Licenciatura). Universidad de Ciencias y Artes de Chiapas, México.

Juárez, T. E. (2002). Distribución y abundancia del jaguar Panthera onca en la Reserva de la Biósfera La Encrucijada, Chiapas (Tesis de Licenciatura). Instituto Tecnológico Agropecuario de Tuxtepec, Oaxaca, México.

Kasper, C. B., Bastazini, V. A. G., Salvi, J., \& Grillo, H. C. Z. (2008). Trophic ecology and the use of shelters and latrines by the Neotropical otter (Lontra longicaudis) in the Taquari Valley, Southern Brazil. Iheringia. Série Zoologia, 98(4), 469-474.

Kay, S., \& Du Croz, T. (2008). Sun Times (Version 7.1). Recuperado de http://www.aptl72.dsl.pipex.com/ suntimes.htm

Kovach Computing Services. (2011). Software Oriana (Version 4). Recuperado de http://www.kovcomp. co.uk/oriana/oribroc.html

Lira-Torres, I., \& Briones-Salas, M. (2012). Abundancia relativa y patrones de actividad de los mamíferos de los Chimalapas, Oaxaca, México. Acta Zoológica Mexicana (n.s), 28, 566-585.

Lira-Torres, I., Briones-Salas, M., \& Sánchez-Rojas, G. (2014). Abundancia relativa, estructura poblacional, preferencia de hábitat y patrones de actividad del Tapir Centroamericano Tapirus bairdii (Perissodactyla: Tapiridae), en la selva de Los Chimalapas, Oaxaca, México. Revista de Biología Tropical, 62, 1407-1419.

Mackey, A. P., \& Smail, G. (1995). Spatial and temporal variation in litter fall of Avicennia marina (Forssk.) Vierh. in the Brisbane River, Queensland, Australia. Aquatic Botany, 52(1-2), 133-142.

Maffei, L., Cuellar, E., \& Noss, J. (2002). Uso de trampas cámara para la evaluación de mamíferos en el ecotono Chaco-Chiquitanía. Revista boliviana de ecología y conservación ambiental, 11, 55-65.

Mitsch, W., Gosselink, G., Anderson, C., \& Zhang, L. (2009). Wetland ecosystems. New York: John Wiley y Sons Inc.

Monroy-Vilchis, O., \& Mundo, V. (2009). Nicho trófico de la nutria neotropical (Lontra longicaudis) en un ambiente modificado, Temascaltepec, México. Revista mexicana de biodiversidad, 80(3), 801-806.

Monroy-Vilchis, O., Zarco-González, M., Rodríguez-Soto, C., Soria-Díaz, L., \& Urios, V. (2011). Fototrampeo de mamíferos en la Sierra Nanchititla, México: abundancia relativa y patrón de actividad. Revista de Biología Tropical, 59(1), 373-383.
Parks, P. J., \& Bonifaz, M. (1994). Nonsustainable use of and mariculture in Ecuador. Marine Resources Economics, 91, 1-18.

Pérez-Irineo, G., \& Santos-Moreno, A. (2010). Diversidad de una comunidad de mamíferos carnívoros en una selva mediana del noreste de Oaxaca, México. Acta Zoológica Mexicana (n.s), 26, 721-736.

Ramsar. (2013). Manual de la Convención de Ramsar: Guía a la Convención sobre los Humedales (Ramsar, Irán, 1971), 6a edición. Suiza: Secretaría de la Convención de Ramsar. Recuperado de www.ramsar.org/ sites/default/files/documents/library/manual6-2013sp.pdf

R Core Team. (2017). R: A language and environment for statistical computing. Austria: R Foundation for Statistical Computing. Recuperado de https:// www.R-project.org/

Rivadeneyra, E., \& Naranjo, E. (2004). Estimación preliminar del ámbito hogareño de un grupo de pecaríes de collar (Tayassu tajacu) en la Reserva de la Biósfera "La Encrucijada", Chiapas, México. Memoria III Congreso de Estudiantes del Verano de la Investigación, México.

Rivero, M., \& Medellín, R. (2015). Mamíferos del estado de Chiapas. Revista Mexicana de Mastozoología Nueva época, 5(2), 23-37.

Rodríguez-Zúñiga, M. T., Troche-Souza, C., VázquezLule, A. D., Márquez-Mendoza, J. D., Vázquez- Balderas, B., Valderrama-Landeros, L., ... Galindo-Leal, C. (2013). Manglares de México/Extensión, distribución y monitoreo. México: Comisión Nacional para el Conocimiento y Uso de la Biodiversidad.

Romero-Berny, E. I., \& Tovilla-Hernández, C. (2009). Estructura del manglar en el sistema lagunar costero de Carretas-Pereyra, Reserva de la Biosfera La Encrucijada, Chiapas, México. Lacandonia, 3(1), 19-28.

Sanjurjo-Rivera, E., \& Campos-Palacín, P. (2011). Análisis de las actividades económicas en un manglar de usos múltiples. Un estudio de caso en San Blas, Nayarit, México. Estudios sociales, 19(38), 197-220.

SEMARNAT. (2010). Norma Oficial Mexicana NOM-059ECOL-2010. Protección ambiental-especies nativas de México de flora y fauna silvestres. Categorías de riesgo y especificaciones para su inclusión, exclusión o cambio. Lista de especies en riesgo. México: Diario Oficial de la Federación. Recuperado de www. profepa.gob.mx/innovaportal/file/435/1/NOM_059 SEMARNAT_2010.pdf

SEMARNAT. (2014). Politica Nacional de Humedales. México, D.F.

Servín, J., \& Chacón, E. (2005). Coyote. In G. Ceballos \& G. Oliva (Eds.), Los mamíferos silvestres de México (pp. 349-350). México: FCE-CONABIO. 
Sokal, R. R., \& Rohlf, J. F. (1981). Statistical tables. New York: W. H. Freeman Company.

Spalding, M., Kainuma, M., \& Collins, L. (2010). Earthscan. World Mangrove Atlas.

Srbek-Araujo, A. C., \& Chiarello, A. G. (2005). Is camera trapping an efficient method for surveying mammals in neotropical forests? A case study in south-eastern - Brazil. Journal of Tropical Ecology, 21, 121-125.

ten Brink P., Russi D., Farmer A., Badura T., Coates D., Förster J., Kumar R., \& Davidson, N. (2013). La Economía de los Ecosistemas y la Biodiversidad relativa al agua y los humedales. Resumen ejecutivo. Instituto de Política Medioambiental Europea (IEEP) y Secretaría de Ramsar, 2013. Recuperado de www. ramsar.org/sites/default/files/documents/library/teeb_ waterwetlands_execsum_2013-sp.pdf

Tovilla-Hernández, C. (2007). Estudio diagnóstico: situación actual de los manglares en la Reserva de Biosfera La Encrucijada y su área de influencia Chiapas, México: transferencia y sociabilización del conocimiento. México: El Colegio de la Frontera Sur, Tapachula, Chiapas.

Torres-Cristiani, L. (2010). Análisis genético del pecarí de collar (Tayassu tajacu) en el Sureste de México.
(Tesis de Maestría). Colegio de la Frontera Sur, Chiapas, México.

Troche-Souza, C., Rodríguez-Zúñiga, M. T., VelázquezSalazar, S., Valderrama-Landeros, L., Villeda-Chávez, E., Alcántara-Maya, A., ... Ressl, R. 2016. Manglares de México: extensión, distribución y monitoreo (1970/1980-2015). México: Comisión Nacional para el Conocimiento y Uso de la Biodiversidad.

Valenzuela, D., \& Ceballos, G. (2000). Habitat selection, home range and activity of the White nosed coati (Nasua nasua) in a Mexican Tropical Dry Forest. Journal of Mammalogy, 81, 810-819.

Valenzuela, D., \& D. W. Macdonald. (2002). Home-range use by white-nosed coatis (Nasua narica): Limited water and a test of the resource dispersion hypothesis. Journal of Zoology, 258, 247-256.

Van der Valk, A. G. (2006). The biology of freshwater wetlands. Oxford, United Kingdom: Oxford University Press.

Van Schaik, C. P., \& Griffiths, M. (1996). Activity periods of Indonesian rain forest mammals. Biotropica, 28 , 105-112.

Zar, J. H. (1998). Biostatistical Analysis (4th ed.). Upper Saddle River, N.J: Pearson Prentice-Hall. 